\title{
Existence and dimension of the set of mild solutions to semilinear fractional differential inclusions
}

Ravi P Agarwal ${ }^{1,2}$, Bashir Ahmad $^{2}$, Ahmad Alsaedi $^{2}$ and Naseer Shahzad ${ }^{2 *}$

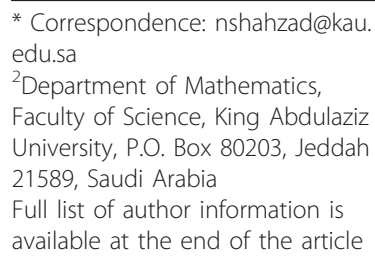

* Correspondence: nshahzad@kau. edu.sa

${ }^{2}$ Department of Mathematics, Faculty of Science, King Abdulaziz University, P.O. Box 80203, Jeddah 21589, Saudi Arabia

Full list of author information is available at the end of the article

\begin{abstract}
This article studies the existence and dimension of the set for mild solutions of semilinear fractional differential inclusions. We recall and prove some new results on multivalued maps to establish our main results.

MSC 2010: 34A12; 34A40.

Keywords: nonlocal problem, fractional differential inclusions, topological dimension, mild solution, fixed point theorems
\end{abstract}

\section{Introduction}

The study of fractional calculus (differentiation and integration of arbitrary order) has emerged as an important and popular field of research. It is mainly due to the extensive application of fractional differential equations in many engineering and scientific disciplines such as physics, chemistry, biology, economics, control theory, signal and image processing, biophysics, blood flow phenomena, aerodynamics, fitting of experimental data, etc., [1-7]. Fractional derivatives are also regarded as an excellent tool for the description of memory and hereditary properties of various materials and processes [8]. Owing to these characteristics of fractional derivatives, fractional-order models are considered to be more realistic and practical than the classical integer-order models, in which such effects are not taken into account. A variety of results on initial and boundary value problems of fractional differential equations, ranging from the theoretical aspects of existence and uniqueness of solutions to the analytic and numerical methods for finding solutions, have appeared in the literature, for instance, see [9-20] and references therein.

Differential inclusions arise in the mathematical modeling of certain problems in economics, optimal control, etc., and are widely studied by many authors, see [21-23] and the references therein. For some recent development on differential inclusions of fractional order, we refer the reader to the references [24-29].

In this article, we discuss the existence and dimension of the set for the mild solutions of the following inclusion problem

$$
\left\{\begin{array}{c}
{ }^{c} D^{q} x(t) \in A x(t)+F(t, x(t)), t \in[0, T], 0<q \leq 1, T>0, \\
x(0)+g(x)=x_{0}, x_{0} \in \mathbb{R}^{n},
\end{array}\right.
$$

(c) 2012 Agarwal et al; licensee Springer. This is an Open Access article distributed under the terms of the Creative Commons Attribution License (http://creativecommons.org/licenses/by/2.0), which permits unrestricted use, distribution, and reproduction in any medium, provided the original work is properly cited. 
where ${ }^{c} D^{q}$ denotes the Caputo fractional derivative of order $q, A$ is a sectorial operator on $\mathbb{R}^{n}$, $g: C\left([0, T], \mathbb{R}^{n}\right) \rightarrow \mathbb{R}^{n}$, and $F:[0, T] \times \mathbb{R}^{n} \rightarrow P\left(\mathbb{R}^{n}\right)$, where $P\left(\mathbb{R}^{n}\right)$ is the family of all nonempty subsets of $\mathbb{R}^{n}$.

\section{Background material}

Let us recall some basic definitions on multi-valued maps (for details, see [30,31]).

Let $(X, d)$ be a metric space. Define $P(X)=\{Y \subseteq X: Y \neq \varnothing\}, P_{c l}(X)=\{Y \in P(X): Y$ is closed $\}, P_{b}(X)=\{Y \in P(X): Y$ is bounded $\}, P_{b}, c l(X)=\{Y \in P(X): Y$ is closed and bounded $\}$ and $P_{c p}(X)=\{Y \in P(X): Y$ is compact $\}$ :

Consider $H: P(X) \times P(X) \rightarrow \mathbb{R} \cup\{\infty\}$ given by

$$
H(A, B)=\max \left\{\sup _{a \in A} d(a, B), \sup _{b \in B} d(b, A)\right\},
$$

where $d(a, B)=\inf _{b \downarrow} d(a, b) . H$ is the (generalized) Pompeiu-Hausdorff functional. It is known that $\left(P_{b, c l}(X), H\right)$ is a metric space and $\left(P_{c l}(X), H\right)$ is a generalized metric space (see [30]).

A multivalued operator $\Omega: X \rightarrow P_{c l}(X)$ is called a $k$-contraction if there exists $0<k$ $<1$ such that

$$
H(\Omega(x), \Omega(y)) \leq k d(x, y) \text { for each } x, y \in X .
$$

Let $C$ be a subset of $X$. A multi-valued map $\Omega: C \rightarrow P(X)$ is called upper semi-continuous (u.s.c.) if $\{x \in C: \Omega(x) \subset V\}$ is open in $C$ whenever $V \subset X$ is open. $\Omega$ is called lower semi-continuous (1.s.c.) if the set $\{y \in C: \Omega(y) \cap V \neq \varnothing\}$ is open for any open set $V \subset X . \Omega$ is called continuous if it is both l.s.c. and u.s.c. It is known that $\Omega: X \rightarrow P_{c p}$ $(X)$ is continuous on $X$ if and only if $\Omega$ is continuous on $X$ with respect to Hausdorff metric. Also, if $\Omega: X \rightarrow P_{c p}(X)$ is a $k$-contraction, then $\Omega$ is continuous with respect to Hausdorff metric. $\Omega$ is said to be completely continuous if $\Omega(\mathbb{B})$ is relatively compact for every $\mathbb{B} \in P_{b}(C)$. A mapping $f: C \rightarrow X$ is called a selection of $\Omega$ if $f(x) \in \Omega(x)$ for every $x \in C$. We say that the mapping $\Omega$ has a fixed point if there is $x \in X$ such that $x \in \Omega(x)$. The fixed points set of the multivalued operator $F$ will be denoted by Fix $(\Omega)$. A multivalued map $\Omega:[0, T] \rightarrow P_{c l}\left(\mathbb{R}^{n}\right)$ is said to be measurable if for every $y \in$ $\mathbb{R}^{n}$, the function

$$
t \mapsto d(y, \Omega(t))=\inf \{|| y-z \|: z \in \Omega(t)\}
$$

is measurable.

Let $\left.\mathfrak{C}\left([0, T], \mathbb{R}^{n}\right]\right)$ denotes the Banach space of continuous functions from $[0, T]$ into $\mathbb{R}^{n}$ with the norm $\mathbb{\|} x \mathbb{W}_{\infty}=\sup _{t \in[0, T]} \mathbb{\|} x(t) \mathbb{}$. Let $L^{1}\left([0, T], \mathbb{R}^{n}\right)$ be the Banach space of measurable functions $x:[0, T] \rightarrow \mathbb{R}^{n}$ which are Lebesgue integrable and normed by

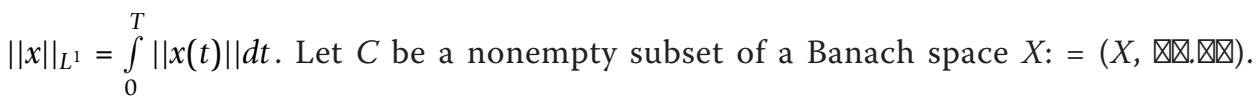
Define $P_{c, c l}(C)=\{Y \in P(C): Y$ is convex and closed $\}$, and $P_{c, c p}(C)=\{Y \in P(C): Y$ is compact and convex\}.

Let us recall some definitions on fractional calculus. For more details, we refer to $[1,4]$.

Definition 2.1. For at least $n$-times continuously differentiable function $g:[0, \infty) \rightarrow$ $\mathbb{R}$, the Caputo derivative of fractional order $q$ is defined as 


$$
{ }^{c} D^{q} g(t)=\frac{1}{\Gamma(n-q)} \int_{0}^{t}(t-s)^{n-q-1} g^{(n)}(s) d s, n-1<q<n, n=[q]+1, q>0,
$$

where $[q]$ denotes the integer part of the real number $q$ and $\Gamma$ denotes the gamma function.

Definition 2.2. The Riemann-Liouville fractional integral of order $q$ for a continuous function $g$ is defined as

$$
I^{q} g(t)=\frac{1}{\Gamma(q)} \int_{0}^{t} \frac{g(s)}{(t-s)^{1-q}} d s, q>0,
$$

provided the right-hand side is pointwise defined on $(0, \infty)$.

\section{Main results}

Definition 3.1. Let $A$ : $D \subseteq \mathbb{R}^{n} \rightarrow \mathbb{R}^{n}$ be a closed linear operator. $A$ is said to be a sectorial operator of type $(M, \theta, \mu)$ if there exist $0<\theta<\pi / 2, M>0, \mu \in \mathbb{R}$ such that the resolvent of $A$ exists outside the sector

$$
\mu+S_{\theta}=\{\mu+\lambda: \lambda \in \mathbb{C}, \operatorname{Arg}(-\lambda) \mid<\theta\}
$$

with

$$
\left\|(\lambda I-A)^{-1}\right\| \leq \frac{M}{|\lambda-\mu|}, \lambda \notin \mu+S_{\theta} .
$$

To define mild solutions for (1), we consider the Cauchy problem

$$
\left\{\begin{array}{c}
{ }^{c} D^{q} x(t)=A x(t)+\sigma(t), t \in[0, T], 0<q \leq 1, T>0 \\
x(0)+g(x)=x_{0}, x_{0} \in \mathbb{R}^{n}
\end{array}\right.
$$

where $\sigma:[0, T] \rightarrow \mathbb{R}^{n}$.

The following lemma is discussed in [32]. However, for the sake of completeness, we outline its proof here.

Lemma 3.2. Let $A$ be a sectorial operator of type $(M, \theta, \mu)$. If $\sigma$ satisfies a uniform Hölder condition with exponent $\beta \in(0,1]$, then the unique solution of the Cauchy problem (2) is given by

$$
x(t)=S_{q}(t)\left(x_{0}-g(x)\right)+\int_{0}^{t} T_{q}(t-s) \sigma(s) d s,
$$

where

$$
S_{q}(t)=\frac{1}{2 \pi i} \int_{\mathcal{P}} e^{\lambda t} \lambda^{q-1} R\left(\lambda^{q}, A\right) d \lambda, T_{q}(t)=\frac{1}{2 \pi i} \int_{\mathcal{P}} e^{\lambda t} R\left(\lambda^{q}, A\right) d \lambda,
$$

where $\mathcal{P}$ is a suitable path such that $\lambda^{q} \notin \mu+S_{\theta}$ for $\lambda \in \mathcal{P}$ and $R\left(\lambda^{q}, A\right)=\left(\lambda^{q} I-A\right)^{-1}$.

Proof. Taking inverse Laplace transform of (2), we get

$$
\lambda^{q}(\mathcal{L} x)(\lambda)-\lambda^{q-1}\left(x_{0}-g(x)\right)=A(\mathcal{L} x)(\lambda)+(\mathcal{L} \sigma)(\lambda),
$$


which implies that

$$
(\mathcal{L} x)(\lambda)=\lambda^{q-1}\left(\lambda^{q} I-A\right)^{-1}\left(x_{0}-g(x)\right)+\left(\lambda^{q} I-A\right)^{-1}(\mathcal{L} \sigma)(\lambda) .
$$

By taking inverse Laplace transform of (4), we obtain (3). This completes the proof. It has been shown in [32] that

$$
\sup _{t \in[0, T]}\left\|S_{q}(t)\right\|=\widetilde{M_{S}},\left\|T_{q}\right\| \leq t^{q-1} \widetilde{M_{T}}
$$

with $\widetilde{M_{S}}=\sup _{t \in[0, T]}\left\|S_{q}(t)\right\|_{L\left(\mathbb{R}^{n}\right)}$, and $\widetilde{M_{T}}=\sup _{t \in[0, T]} C e^{\mu t}\left(1+t^{1-q}\right)$, where $L\left(\mathbb{R}^{n}\right)$ is the Banach space of bounded linear operators from $\mathbb{R}^{n}$ into $\mathbb{R}^{n}$ equipped with natural topology and $C, \mu$ are appropriate constants (for more details see Equation (3.1) in [32]).

Remark 3.3. The definition of the mild solution used in [33] is not appropriate as it does not correspond to the classical case due to the failure of the Leibniz product rule for the Caputo fractional derivative. For more details, see [32].

Definition 3.4. A function $\left.x \in \mathbb{C}\left([0, T], \mathbb{R}^{n}\right]\right)$ is a mild solution of the problem (1) if there exists a function $f \in L,{ }^{1}\left([0, T], \mathbb{R}^{n}\right)$ such that $f(t) \in F(t, x(t))$ a.e. on $[0, T]$ and

$$
x(t)=S_{q}(t)\left(x_{0}-g(x)\right)+\int_{0}^{t} T_{q}(t-s) f(s) d s .
$$

Let $\mathcal{S}_{x_{0}}([0, \alpha])$ denotes the set of all solutions of (1) on the interval $[0, \alpha]$, where 0 $<\alpha \leq T$.

To prove the existence of solutions for (1), we need the following lemma due to Nadler and Covitz [34].

Lemma 3.5. Let $(X, d)$ be a complete metric space. If $\Omega: X \rightarrow P_{c l}(X)$ is a $k$-contraction, then $\operatorname{Fix}(\Omega) \neq \varnothing$.

Theorem 3.6. Assume that

$\left(\mathbf{A}_{\mathbf{1}}\right) F:[0, T] \times \mathbb{R}^{n} \rightarrow P_{c p}\left(\mathbb{R}^{n}\right)$ is such that $F(., x):[0, T] \rightarrow P_{c, c p}\left(\mathbb{R}^{n}\right)$ is measurable for each $x \in \mathbb{R}^{n}$;

$\left(\mathbf{A}_{2}\right) H(F(t, x), F(t, \bar{x})) \leq \kappa_{1}(t)\|x-\bar{x}\|$ for almost all $t \in[0, T]$ and $x, \bar{x} \in \mathbb{R}^{n}$ with $\kappa_{1}$

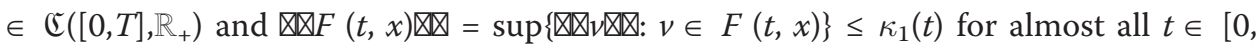
$T$ ] and $x \in \mathbb{R}^{n}$;

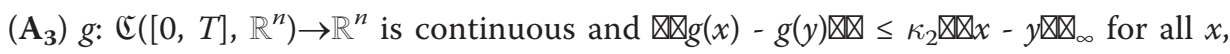
$y \in \mathfrak{C}\left([0, T], \mathbb{R}^{n}\right)$ with some $\kappa_{2}>0$.

Then the Cauchy problem (1) has at least one solution on $[0, T]$ if

$$
\left(\widetilde{M_{S} \kappa_{2}}+\widetilde{M_{T}}\left(T^{q} / q\right)\left\|\kappa_{1}\right\|_{\infty}\right)<1
$$

$\left(\widetilde{M_{S}}\right.$ and $\widetilde{M_{T}}$ are given by (5)).

Proof. For each $y \in \mathbb{C}\left([0, T], \mathbb{R}^{n}\right)$, define the set of selections of $F$ by

$$
S_{F, y}:=\left\{v \in L^{1}\left([0, T], \mathbb{R}^{n}\right): v(t) \in F(t, y(t)) \text { for a.e. } t \in[0, T]\right\} .
$$

Observe that by the assumptions $\left(\mathbf{A}_{\mathbf{1}}\right)$ and $\left(\mathbf{A}_{\mathbf{2}}\right), F(t, x(t))$ is measurable and has a measurable selection $v(t)$ (see [[35], Theorem III.6]). Also $\kappa_{1} \in \mathfrak{C}\left([0, T], \mathbb{R}_{+}\right)$and

$$
\|v(t)\| \leq\|F(t, x(t))\| \leq \kappa_{1}(t) .
$$


Thus the set $S_{F, x}$ is nonempty for each $x \in \mathfrak{C}\left([0, T], \mathbb{R}^{n}\right)$. Let us define an operator $\Omega$ by

$$
\Omega(x)=\left\{h \in \mathfrak{C}\left([0, T], \mathbb{R}^{n}\right): h(t)=S_{q}(t)\left(x_{0}-g(x)\right)+\int_{0}^{t} T_{q}(t-s) f(s) d s, f \in S_{F, x}\right\},
$$

and show that it satisfies the conditions of Lemma 3.5. As a first step, we show that $\Omega(x) \in P_{c l}\left(\mathbb{C}\left([0, T], \mathbb{R}^{n}\right)\right)$ for each $x \in \mathbb{C}\left([0, T], \mathbb{R}^{n}\right)$. Let $\left\{u_{n}\right\}_{n \geq 0} \in \Omega(x)$ be such that $u_{n}$ $\rightarrow u(n \rightarrow \infty)$ in $\mathbb{C}\left([0, T], \mathbb{R}^{n}\right)$. Then $u \in \mathbb{C}\left([0, T], \mathbb{R}^{n}\right)$ and there exists $v_{n} \in S_{F, x}$ such that, for each $t \in[0, T]$,

$$
u_{n}(t)=S_{q}(t)\left(x_{0}-g(x)\right)+\int_{0}^{t} T_{q}(t-s) v_{n}(s) d s
$$

As $F$ has compact values, we pass to a subsequence to obtain that $v_{n}$ converges to $v$ in $\mathfrak{C}\left([0, T], \mathbb{R}^{n}\right)$. Thus, $v \in S_{F, x}$ and for each $t \in[0, T]$,

$$
u_{n}(t) \rightarrow u(t)=v(t)
$$

Hence $u \in \Omega(x)$.

Next we show that there exists a $\gamma \in(0,1)$ such that

$$
H(\Omega(x), \Omega(\bar{x})) \leq \gamma\|x-\bar{x}\|_{\infty} \text { for each } x, \bar{x} \in \mathfrak{C}\left([0, T], \mathbb{R}^{n}\right) .
$$

Let $x, \bar{x} \in \mathfrak{C}\left([0, T], \mathbb{R}^{n}\right)$ and $h_{1} \in \Omega(x)$. Then there exists $v_{1}(t) \in S_{F, x}$ such that, for each $t \in[0, T]$,

$$
h_{1}(t)=S_{q}(t)\left(x_{0}-g(x)\right)+\int_{0}^{t} T_{q}(t-s) v_{1}(s) d s .
$$

By $\left(\mathbf{A}_{2}\right)$, we have

$$
H(F(t, x), F(t, \bar{x})) \leq \kappa_{1}(t)\|x(t)-\bar{x}(t)\| .
$$

So, there exists $w \in F(t, \bar{x}(t))$ such that

$$
\left\|v_{1}(t)-w\right\| \leq \kappa_{1}(t)\|x(t)-\bar{x}(t)\|, \quad t \in[0, T] .
$$

Define $V:[0, T] \rightarrow P\left(\mathbb{R}^{n}\right)$ by

$$
\left.V(t)=\left\{w \in \mathbb{R}^{n}:\left\|v_{1}(t)-w\right\| \leq \kappa_{1}(t)\|x(t)-\bar{x}(t)\|\right)\right\}
$$

Since the nonempty closed valued operator $V(t) \cap F(t, \bar{x}(t))$ is measurable [[35], Proposition III.4], there exists a function $v_{2}(t)$ which is a measurable selection for $V(t) \cap F(t, \bar{x}(t))$. So $v_{2}(t) \in F(t, \bar{x}(t))$ and for each $t \in\left[\begin{array}{ll}0, & T\end{array}\right]$, we have $\left\|v_{1}(t)-v_{2}(t)\right\| \leq \kappa_{1}(t)\|x(t)-\bar{x}(t)\|$. For each $t \in[0, T]$, let us define

$$
h_{2}(t)=S_{q}(t)\left(x_{0}-g(x)\right)+\int_{0}^{t} T_{q}(t-s) v_{2}(s) d s
$$

Thus

$$
\left.\left\|h_{1}(t)-h_{2}(t)\right\| \leq\left\|S_{q}(t)\right\| \| g(x)-g(\bar{x})\right)\left\|+\int_{0}^{t}\right\| T_{q}(s-t)\|\| v_{1}(s)-v_{2}(s) \| d s .
$$


In view of (5), it follows that

$$
\begin{aligned}
\left\|h_{1}-h_{2}\right\|_{\infty} & \leq \widetilde{M_{S}} \kappa_{2}|| x-\bar{x}\left\|_{\infty}+\widetilde{M}_{T}\left(T^{q} / q\right)\right\| \kappa_{1}\left\|_{\infty}\right\| x-\bar{x} \|_{\infty} \\
& =\left(\widetilde{M_{S}} \kappa_{2}+\widetilde{M_{T}}\left(T^{q} / q\right)\left\|\kappa_{1}\right\|_{\infty}\right)\|x-\bar{x}\|_{\infty} .
\end{aligned}
$$

Analogously, interchanging the roles of $x$ and $\bar{x}$, we obtain

$H(\Omega(x), \Omega(\bar{x})) \leq \gamma\|x-\bar{x}\|_{\infty}$ for each $x, \bar{x} \in \mathfrak{C}\left([0, T], \mathbb{R}^{n}\right)$,

where $\gamma=\left(\widetilde{M}_{S} \kappa_{2}+\widetilde{M}_{T}\left(T^{q} / q\right)\left\|\kappa_{1}\right\|_{\infty}\right)<1$. Since $\Omega$ is a contraction, it follows by Lemma 3.5 that $\Omega$ has a fixed point $x$ which is a solution of (1). This completes the proof.

Lemma 3.7. Let $F:[0, T] \times \mathbb{R}^{n} \rightarrow P_{c, c p}\left(\mathbb{R}^{n}\right)$ satisfy $\left(\mathbf{A}_{\mathbf{1}}\right),\left(\mathbf{A}_{\mathbf{2}}\right)$, and $\left(\mathbf{A}_{\mathbf{3}}\right)$ and suppose that $\Omega: \mathfrak{C}\left([0, T], \mathbb{R}^{n}\right) \rightarrow P\left(\mathfrak{C}\left([0, T], \mathbb{R}^{n}\right)\right)$ is defined by

$$
\Omega(x)=\left\{h \in \mathfrak{C}\left([0, T], \mathbb{R}^{n}\right): h(t)=x_{0}-g(x)+\int_{0}^{t} \frac{(t-s)^{q-1}}{\Gamma(q)} f(s) d s, f \in S_{F, x}\right\}
$$

Then $\Omega(x) \in P_{c, c p}\left(\mathfrak{C}\left([0, T], \mathbb{R}^{n}\right)\right)$ for each $x \in \mathfrak{C}\left([0, T], \mathbb{R}^{n}\right)$.

Proof. First we show that $\Omega(x)$ is convex for each $x \in\left([0, T], \mathbb{R}^{n}\right)$. For that, let $h_{1}$, $h_{2} \in \Omega(x)$. Then there exist $f_{1}, f_{2} \in S_{F, x}$ such that for each $t \in[0, T]$, we have

$$
h_{i}(t)=S_{q}(t)\left(x_{0}-g(x)\right)+\int_{0}^{t} T_{q}(t-s) f_{i}(s) d s, \quad i=1,2 .
$$

Let $0 \leq \lambda \leq 1$. Then, for each $t \in[0, T]$, we have

$$
\begin{aligned}
& {\left[\lambda h_{1}+(1-\lambda) h_{2}\right](t)} \\
& \quad=S_{q}(t)\left(x_{0}-g(x)\right)+\int_{0}^{t} T_{q}(t-s)\left[\lambda f_{1}(s)+(1-\lambda) f_{2}(s)\right] d s .
\end{aligned}
$$

Since $S_{F, x}$ is convex ( $F$ has convex values), therefore it follows that $\lambda h_{1}+(1-\lambda) h_{2} \in$ $\Omega(x)$. Next, we show that $\Omega$ maps bounded sets into bounded sets in $\mathbb{C}\left([0, T], \mathbb{R}^{n}\right)$. For a positive number $r$, let $B_{r}=\left\{x \in \mathfrak{C}\left([0, T], \mathbb{R}^{n}\right): \mathbb{W} x \mathbb{\bigotimes}_{\infty} \leq r\right\}$ be a bounded set in $\mathfrak{C}([0, T]$, $\left.\mathbb{R}^{n}\right)$. Then, for each $h \in \Omega(x), x \in B_{r}$, there exists $f \in S_{F, x}$ such that

$$
h(t)=S_{q}(t)\left(x_{0}-g(x)\right)+\int_{0}^{t} T_{q}(t-s) f(s) d s
$$

and in view of $\left(H_{1}\right)$, we have

$$
\begin{aligned}
\|h(t)\| & \leq\left|S_{q}(t)\right|\left(\left\|x_{0}\right\|+\sup _{x \in B_{r}}\|g(x)\|\right)+\int_{0}^{t}\left|T_{q}(t-s)\right|\|f(s)\| d s \\
& \leq\left|S_{q}(t)\right|\left(\left\|x_{0}\right\|+\sup _{x \in B_{r}}\|g(x)\|\right)+\int_{0}^{T}\left|T_{q}(t-s)\right| \kappa_{1}(s) d s .
\end{aligned}
$$


Thus,

$$
\|h\|_{\infty} \leq\left|S_{q}(t)\right|\left(|| x_{0}\left\|+\sup _{x \in B_{r}}\right\| g(x)||\right)+\int_{0}^{T}\left|T_{q}(t-s)\right| \kappa_{1}(s) d s .
$$

Now we show that $\Omega$ maps bounded sets into equicontinuous sets in $\mathbb{C}\left([0, T], \mathbb{R}^{n}\right)$. Let $t^{\prime}, t^{\prime \prime} \in[0, T]$ with $t^{\prime}<t^{\prime \prime}$ and $x \in B_{r}$, where $B_{r}$ is a bounded set in $\mathbb{C}\left([0, T], \mathbb{R}^{n}\right)$. For each $h \in \Omega(x)$, we obtain

$$
\begin{aligned}
& \left\|h\left(t^{\prime \prime}\right)-h\left(t^{\prime}\right)\right\| \\
\leq & \left\|\left(S_{q}\left(t^{\prime \prime}\right)-S_{q}\left(t^{\prime}\right)\right)\left(x_{0}-g(x)\right)\right\|+\left\|\int_{0}^{t^{\prime \prime}} T_{q}\left(t^{\prime \prime}-s\right) f(s) d s-\int_{0}^{t^{\prime}} T_{q}\left(t^{\prime}-s\right) f(s) d s\right\| \\
\leq & \left\|\left(S_{q}\left(t^{\prime \prime}\right)-S_{q}\left(t^{\prime}\right)\right)\left(x_{0}-g(x)\right)\right\|+\left\|\int_{0}^{t^{\prime}}\left[T_{q}\left(t^{\prime \prime}-s\right)-T_{q}\left(t^{\prime}-s\right)\right] \kappa_{1}(s) d s\right\| \\
& +\left\|\int_{t^{\prime}}^{t^{\prime \prime}} T_{q}\left(t^{\prime \prime}-s\right) \kappa_{1}(s) d s\right\| .
\end{aligned}
$$

Obviously the right-hand side of the above inequality tends to zero independently of $x \in B_{r^{\prime}}$ as $t^{\prime \prime}-t^{\prime} \rightarrow 0$. By the Arzela-Ascoli Theorem, $\Omega: \mathfrak{C}\left([0, T], \mathbb{R}^{n}\right) \rightarrow P\left(\mathbb{C}\left([0, T], \mathbb{R}^{n}\right)\right)$ is completely continuous. As in Theorem 3.6, $\Omega$ is closed-valued. Consequently, $\Omega(x)$ $\in P_{c, c p}\left(\mathbb{C}\left([0, T], \mathbb{R}^{n}\right)\right)$ for each $x \in \mathbb{C}\left([0, T], \mathbb{R}^{n}\right)$.

For $0<\alpha \leq T$, let us consider the operator

$$
\Omega(x)=\left\{h \in \mathfrak{C}\left([0, \alpha], \mathbb{R}^{n}\right): h(t)=S_{q}(t)\left(x_{0}-g(x)\right)+\int_{0}^{t} T_{q}(t-s) f(s) d s, f \in S_{F, x}\right\} .
$$

It is well-known that $\operatorname{Fix}(\Omega)=\mathcal{S}_{x_{0}}([0, \alpha])$ and, in view of Theorem 3.6, it is nonempty for each $0<\alpha \leq T$.

The following results are useful in the sequel.

Lemma 3.8 (Dzedzej and Gelman [36]) Let $F:[0, \alpha] \rightarrow P_{c, c p}\left(\mathbb{R}^{n}\right)$ be a measurable map such that the Lebesgue measure $\mu$ of the set $\{t \operatorname{dim} F(t)<1\}$ is zero. Then there are arbitrarily many linearly independent measurable selections $x_{1}(\cdot), x_{2}(\cdot), \ldots, x_{m}(\cdot)$ of $F$.

Lemma 3.9. (Dzedzej and Gelman [36]) (see also, $[29,37]$ for general versions) Let $C$ be a nonempty closed convex subset of a Banach space $X$. Suppose that $\Omega: C \rightarrow P_{c, c p}$ $(C)$ is a $k$-contraction. If $f: C \rightarrow C$ is a continuous selection of $\Omega$, then $\operatorname{Fix}(f)$ is nonempty.

Lemma 3.10. (Michael's selection theorem) [38] Let $C$ be a metric space, $X$ be a Banach space and $\Omega: C \rightarrow P_{c, c l}(C)$ a lower semicontinuous map. Then there exists a continuous selection $f: C \rightarrow X$ of $\Omega$.

Lemma 3.11. (Saint Raymond [39]) Let $K$ be a compact metric space with $\operatorname{dim} K<n$, $X$ a Banach space and $\Omega: K \rightarrow P_{c, c p}(X)$ a lower semicontinuous map such that $0 \in$ $\Omega(x)$ and $\operatorname{dim} \Omega(x) \geq n$ for every $x \in K$. Then there exists a continuous selection $f$ of $\Omega$ such that $f(x) \neq 0$ for each $x \in K$.

Theorem 3.12. Let $F:[0, \alpha] \times \mathbb{R}^{n} \rightarrow P_{c, c p}\left(\mathbb{R}^{n}\right)$ satisfy $\left(\mathbf{A}_{1}\right),\left(\mathbf{A}_{2}\right)$, and $\left(\mathbf{A}_{3}\right)$ and suppose that the Lebesgue measure $\mu$ of the set $\left\{t: \operatorname{dim} F(t, x)<1\right.$ for some $\left.x \in \mathbb{R}^{n}\right\}$ is 
zero. Then for each $\alpha, 0<\alpha<\min \left\{\left(\frac{\left(1-\widetilde{M}_{S} \kappa_{2}\right) q}{\widetilde{M}_{T\left\|\kappa_{1}\right\|_{\infty}}}\right)^{\frac{1}{q}}, T\right\}$, the set $\mathcal{S}_{x_{0}}([0, \alpha])$ of solutions of (1) has an infinite dimension for any $x_{0}$.

Proof. Define the operator $\Omega$ by

$$
\Omega(x)=\left\{h \in \mathfrak{C}\left([0, \alpha], \mathbb{R}^{n}\right): h(t)=S_{q}(t)\left(x_{0}-g(x)\right)+\int_{0}^{t} T_{q}(t-s) f(s) d s, f \in S_{F, x}\right\} .
$$

Then by Lemma 3.7, $\left.\Omega(x) \in P_{c, c p}\left(\mathbb{C}([0, \alpha]), \mathbb{R}^{n}\right)\right)$ for each $x \in \mathbb{C}\left([0, \alpha], \mathbb{R}^{n}\right)$ and as in the proof of Theorem 3.6, it is a contraction if $\left(\widetilde{M_{S} \kappa_{2}}+\widetilde{M_{T}}\left(\alpha^{q} / q\right)\left\|\kappa_{1}\right\|_{\infty}\right)<1$ or $\alpha<\left(\frac{\left(1-\tilde{M}_{\left.s \kappa_{2}\right) q}\right.}{\tilde{M}_{T\left\|\kappa_{1}\right\|_{\infty}}}\right)^{\frac{1}{q}}$. We shall show that $\operatorname{dim} \Omega(x) \geq m$ for any $x \in \mathbb{C}\left([0, \alpha], \mathbb{R}^{n}\right)$ and arbitrary $m \in \mathbb{N}$. Consider $G(t)=F(t, x(t))$. By Lemma 3.8, there exist linearly independent measurable selections $x_{1}(),. x_{2}(),. ., ., x_{m}($.$) of G$. Set $y_{i}(t)=S_{q}(t)\left(x_{0}-g(x)\right)+\int_{0}^{t} T_{q}(t-s) x_{i}(s) d s \in \Omega(x)$. Assume that $\sum_{i=1}^{m} a_{i} y_{i}(t)=0$ a.e. in $[0, \alpha]$. Taking Caputo derivatives a.e. in $[0, \alpha]$, we have $\sum_{i=1}^{m} a_{i} x_{i}(t)=0$ a.e. in $[0$, $\alpha]$ and hence $a_{i}=0$ for all $i$. As a result, $y_{i}($.$) are linearly independent. Thus \Omega(x)$ contains an $m$-dimensional simplex. So $\operatorname{dim} \Omega(x) \geq m$. As in Theorem 3.6, $\operatorname{Fix}(\Omega)$ is nonempty. Since $\Omega$ is condensing with respect to the Hausdorff measure of noncompactness $\chi[40]$ and $\operatorname{Fix}(\Omega) \subset \Omega(\operatorname{Fix}(\Omega))$, we have

$$
\mathcal{X}(\operatorname{Fix}(\Omega)) \leq \mathcal{X}(\Omega(\operatorname{Fix}(\Omega))) .
$$

This implies that $\operatorname{Fix}(\Omega)$ is compact. Consider the map $I-\Omega$ : $\operatorname{Fix}(\Omega) \rightarrow P_{c, c p}\left(\mathbb{R}^{n}\right)$, where $I$ is the identity operator. Assume that $\operatorname{dim} \operatorname{Fix}(\Omega)<n$. Then, by Lemma 3.11, there is a continuous selection $g$ of $I-\Omega$ such that $g(x) \neq 0$ for each $x \in \operatorname{Fix}(\Omega)$. This implies that there exists a continuous selection $h$ of $F: \operatorname{Fix}(F) \rightarrow P_{c, c p}\left(\mathbb{R}^{n}\right)$ without fixed points. Define $T: \mathbb{R}^{n} \rightarrow P_{c, c p}\left(\mathbb{R}^{n}\right)$ by

$$
T(x)=\left\{\begin{array}{c}
\Omega(x), \quad x \in \mathbb{R}^{n} \backslash \text { Fix }(\Omega) \\
h(x), \quad x \in \text { Fix }(\Omega)
\end{array}\right.
$$

Since $T$ is lower semicontinuous, Michael's selection result (Lemma 3.10) guarantees that $T$ admits a continuous selection $f: \mathbb{R}^{n} \rightarrow \mathbb{R}^{n}$. Thus $f: \mathbb{R}^{n} \rightarrow \mathbb{R}^{n}$ is a continuous selection of $\Omega$ with no fixed points and $f=h$ on $\operatorname{Fix}(\Omega)$, which contradicts Lemma 3.9. Consequently, $\operatorname{Fix}(\Omega)=\mathcal{S}_{x_{0}}([0, \alpha])$ is infinite dimensional.

Recall that a metric space $X$ is an AR-space if, whenever it is nonempty closed subset of another metric space $Y$, then there exists a continuous retraction $r: Y \rightarrow X, r(x)=x$ for $x \in X$. In particular, it is contractible (and hence connected).

Lemma 3.13. [41] Let $C$ be a nonempty closed convex subset of a Banach space $X$ and $\Omega$ : $C \rightarrow P_{c, c p}(C)$ a contraction. Then $\operatorname{Fix}(\Omega)$ is a nonempty AR-space.

Theorem 3.12 together with Lemma 4.13 yields the following result. 
Corollary 3.14. Let $F:[0, \alpha] \times \mathbb{R}^{n} \rightarrow P_{c, c p}\left(\mathbb{R}^{n}\right)$ satisfy $\left(\mathbf{A}_{\mathbf{1}}\right),\left(\mathbf{A}_{\mathbf{2}}\right)$, and $\left(\mathbf{A}_{\mathbf{3}}\right)$ and suppose that the Lebesgue measure $\mu$ of the set $\left\{t \operatorname{dim} F(t, x)<1\right.$ for some $\left.x \in \mathbb{R}^{n}\right\}$ is zero. Then for each $\alpha, 0<\alpha<\min \left\{\left(\frac{\left(1-\tilde{M}_{S} \kappa_{2}\right) q}{M_{T \| \kappa_{1}} \|_{\infty}}\right)^{\frac{1}{q}}, T\right\}$, the set $\mathcal{S}_{x_{0}}([0, \alpha])$ of solutions of (1) is an infinite dimensional AR-space.

\section{Acknowledgements}

This project was funded by the Deanship of Scientific Research (DSR), King Abdulaziz University, Jeddah, under grant no. 08/31/Gr. The authors, therefore, acknowledge with thanks DSR technical and financial support. The authors are grateful to the reviewers for their useful comments.

\section{Author details}

'Department of Mathematics, Texas A\&M University-Kingsville, 700 University Blvd., Kingsville, TX 78363-8202, USA ${ }^{2}$ Department of Mathematics, Faculty of Science, King Abdulaziz University, P.O. Box 80203, Jeddah 21589, Saudi Arabia

\section{Authors' contributions}

All authors contributed equally and significantly in writing this article. All authors read and approved the final manuscript.

\section{Competing interests}

The authors declare that they have no competing interests.

Received: 31 January 2012 Accepted: 31 May 2012 Published: 31 May 2012

\section{References}

1. Podlubny, I: Fractional Differential Equations. Academic Press, San Diego (1999)

2. Zaslavsky, GM: Hamiltonian Chaos and Fractional Dynamics. Oxford University Press, Oxford (2005)

3. Magin, RL: Fractional Calculus in Bioengineering. Begell House Publisher, Inc., Connecticut (2006)

4. Kilbas, AA, Srivastava, HM, Trujillo, JJ: Theory and Applications of Fractional Differential Equations, North-Holland Mathematics Studies, 204. Elsevier Science B.V., Amsterdam (2006)

5. Sabatier J, Agrawal OP, Machado JAT (eds.): Advances in Fractional Calculus: Theoretical Developments and Applications in Physics and Engineering. Springer, Dordrecht (2007)

6. Lakshmikantham, V, Leela, S, Vasundhara Devi, J: Theory of Fractional Dynamic Systems. Cambridge Academic Publishers, Cambridge (2009)

7. Baleanu, D, Diethelm, K, Scalas, E, Trujillo, JJ: Fractional calculus models and numerical methods. Series on Complexity, Nonlinearity and Chaos, World Scientific, Boston (2012)

8. Lazarevic, MP, Spasic, AM: Finite-time stability analysis of fractional order time delay systems: Gronwall's approach. Math Comput Model. 49, 475-481 (2009). doi:10.1016/j.mcm.2008.09.011

9. El-Sayed, AMA: Fractional order evolution equations. J Fract Calc. 7, 89-100 (1995)

10. Ahn, W, Mcvinish, R: Fractional differential equations driven by Levy noise. J Appl Math Stochastic Anal. 16, 97-119 (2003). doi:10.1155/S1048953303000078

11. Lakshmikantham, V: Theory of fractional differential equations. Nonlinear Anal. 60(10), 3337-3343 (2008)

12. Agarwal, RP, Belmekki, M, Benchohra, M: A survey on semi-linear differential equations and inclusions involving Riemann-Liouville fractional derivative. Adv Difference Equ 2009, 47 (2009). Art. ID 981728

13. Ahmad, B, Nieto, JJ: Existence of solutions for nonlocal boundary value problems of higher order nonlinear fractional differential equations. Abstr Appl Anal 9 (2009). Art. ID 494720

14. Ahmad, B, Graef, JR: Coupled systems of nonlinear fractional differential equations with nonlocal boundary conditions. Panamer Math J. 19, 29-39 (2009)

15. Ahmad, B, Nieto, JJ: Existence results for a coupled system of nonlinear fractional differential equations with three-point boundary conditions. Comput Math Appl. 58, 1838-1843 (2009). doi:10.1016/j.camwa.2009.07.091

16. N'Guerekata, GM: A Cauchy problem for some fractional abstract differential equation with non local conditions. Nonlinear Anal. 70, 1873-1876 (2009). doi:10.1016/j.na.2008.02.087

17. Agarwal, RP, de Andrade, B, Cuevas, C: On type of periodicity and ergodicity to a class of fractional order differential equations. Adv Diff Equ 2010, 25 (2010). Art. ID 179750

18. dos Santos, JPC, Cuevas, C, Arjunan, M: Existence results for a fractional neutral integro-differential equations with statedependent delay. Comput Math Appl. 62, 1275-1283 (2011). doi:10.1016/j.camwa.2011.03.048

19. Agarwal, RP, Ahmad, B: Existence of solutions for impulsive anti-periodic boundary value problems of fractional semilinear evolution equations. Dyn Contin Discrete Impuls Syst Ser A Math Anal. 18, 457-470 (2011)

20. Ahmad, B, Agarwal, RP: On nonlocal fractional boundary value problems. Dyn Contin Discrete Impuls Syst Ser A Math Anal. 18, 535-544 (2011)

21. Smirnov, GV: Introduction to the Theory of Differential Inclusions. American Mathematical Society, Providence, RI (2002)

22. Li, WS, Chang, YK, Nieto, JJ: Solvability of impulsive neutral evolution differential inclusions with state-dependent delay. Math Comput Model. 49, 1920-1927 (2009). doi:10.1016/j.mcm.2008.12.010 
23. Henderson, J, Ouahab, A: Fractional functional differential inclusions with finite delay. Nonlinear Anal. 70, 2091-2105 (2009). doi:10.1016/j.na.2008.02.111

24. Ouahab, A: Some results for fractional boundary value problem of differential inclusions. Nonlinear Anal. 69, 3877-3896 (2008). doi:10.1016/j.na.2007.10.021

25. Ahmad, B, Otero-Espinar, V: Existence of solutions for fractional differential inclusions with anti-periodic boundary conditions. Bound Value Probl 2009, 11 (2009). Art. ID 625347

26. Ahmad, B, Shahzad, N: A nonlocal boundary value problem for fractional differential inclusions of arbitrary order involving convex and non-convex valued maps. Veitnam J Math. 38, 435-451 (2010)

27. Ahmad, B, Ntouyas, SK: Some existence results for boundary value problems of fractional differential inclusions with non-separated boundary conditions. Electron J Qual Theory Diff Equ. 71, 1-17 (2010)

28. Ahmad, B, Ntouyas, SK: Existence of solutions for fractional differential inclusions with nonlocal strip conditions. Arab J Math Sci (2012). doi:10.1016/j.ajmsc.2012.01.005

29. Agarwal, RP, Ahmad, B, Alsaedi, A, Shahzad, N: Dimension of the solution set for fractional differential inclusions. J Nonlinear Convex Anal (2012, in press). 13

30. Hu, S, Papageorgiou, N: Handbook of Multivalued Analysis, Theory I. Kluwer, Dordrecht. (1997)

31. Dugundji, J, Granas, A: Fixed Point Theory. Springer-Verlag, New York (2005)

32. Shu, $X B$, Lai, YZ, Chen, Y: The existence of mild solutions for impulsive fractional partial differential equations. Nonlinear Anal. 74, 2003-2011 (2011). doi:10.1016/j.na.2010.11.007

33. Jaradat, OK, Al-Omari, A, Momani, S: Existence of the mild solution for fractional semilinear initial value problems. Nonlinear Anal. 69, 3153-3159 (2008). doi:10.1016/..na.2007.09.008

34. Covitz, H, Nadler, SB Jr: Multi-valued contraction mappings in generalized metric spaces. Israel J Math. 8, 5-11 (1970). doi:10.1007/BF02771543

35. Castaing, C, Valadier, M: Convex Analysis and Measurable Multifunctions, Lecture Notes in Mathematics 580. SpringerVerlag, Berlin (1977)

36. Dzedzej, Z, Gelman, BD: Dimension of the solution set for differential inclusions. Demonstratio Math. 26(1), 149-158 (1993)

37. Petrusel, A: Multivalued operators and continuous selections. The fixed points set. Pure Math Appl. 9(1-2), 165-170 (1998)

38. Michael, E: Continuous selections. I Ann Math. 63(2), 361-382 (1956). doi:10.2307/1969615

39. Saint-Raymond, J: Points fixes des multiapplications á valeurs convexes. (French) [Fixed points of multivalued maps with convex values] C. R. Acad Sci Paris Sér I Math. 298, 71-74 (1984)

40. Akhmerov, RR, Kamenskii, MI, Potapov, AS, Rodkina, AE, Sadovskii, BN: Measures of noncompactness and condensing operators. Translated from the 1986 Russian original by A. lacob. Operator Theory: advances and applications, 55. Birkhauser Verlag, Basel (1992)

41. Ricceri, B: Une propriété topologique de l'ensemble des points fixes d'une contraction multivoque valeurs convexes. (French) [A topological property of the set of fixed points of a multivalued contraction with convex values]. Atti Accad Naz Lincei Rend Cl Sci Fis Mat Natur 81(3), 283-286 (1987). (8)

doi:10.1186/1687-1847-2012-74

Cite this article as: Agarwal et al: Existence and dimension of the set of mild solutions to semilinear fractional differential inclusions. Advances in Difference Equations 2012 2012:74.

\section{Submit your manuscript to a SpringerOpen ${ }^{\odot}$ journal and benefit from:}

- Convenient online submission

- Rigorous peer review

- Immediate publication on acceptance

- Open access: articles freely available online

- High visibility within the field

- Retaining the copyright to your article

Submit your next manuscript at $\gg$ springeropen.com 